but achieved this greater duration of sleep by means of naps which were longer than naps taken on working days. ${ }^{5}$

Wakefulness merges into sleep along a continuum, and as we drift towards sleep we fail to perform as efficiently and take longer to respond to something which merits attention. Under five hours of sleep the previous night makes this tendency more marked, ${ }^{6}$ while absolute sleeplessness makes errors frequent on any prolonged task. ${ }^{7}$ Thirty hours without sleep will seriously impair performance on a 25 -minute task requiring continuous observation ${ }^{8}$ and causes intermittent very long reaction times, or " microsleeps." 7 The more prolonged the task the more obtrusive the failures.

Most motorway drivers, even when not short of sleep, have noticed occasions of drowsiness. Even if the eyes are forcibly kept open, extreme imposed monotony can quickly cause sleep, especially if movement is restricted. ${ }^{9}$ The frequent drifts towards drowsiness which accompany monotony are associated with the slow E.E.G. of drowsiness, and it is at moments of E.E.G. slowing that reaction time is very prolonged. ${ }^{10}$ These brief naps are even more likely after alcohol, and an ordinary sleeping-pill, such as quinalbarbitone 200 mg., will increase such lapses during the following day for at least 14 hours after ingestion of the drug. ${ }^{11}$

Napping under appropriate circumstances can help restore us, but a nap at the wheel of a car can be disastrous. Lack of sleep, sedatives, alcohol, warmth, restricted movements, and monotony will increase the liability to a nap. Frequent breaks, with physical activity and other elements of novelty, will help ensure alertness..

\section{Malignant Granuloma}

Malignant granuloma, also called lethal midline granuloma and granuloma gangraenescens, is a relentlessly destructive chronic inflammatory process occurring in the region of the nose. It leads to necrosis of the soft tissues, cartilage, and bone, and by ulceration causes extensive mutilation of the face and eyes. The course of the untreated disease is usually several months, and death occurs from cachexia, haemorrhage, or intercurrent infection. ${ }^{1}$

Two histological varieties have been recognized. In the one first described by J. P. Stewart ${ }^{2}$ there is a heavy pleomorphic cellular infiltration consisting predominantly of lymphocytes, plasma cells, and macrophages in association with extensive necrosis and formation of granulation-tissue. In the second variety, described by $\mathrm{F}$. Wegener, ${ }^{3}$ there is a necrotizing giant-cell granulomatous lesion which usually affects the lungs also. The other features of Wegener's granulomatosis are generalized necrotizing angiitis and glomerulonephritis. ${ }^{45}$ These two varieties are probably not completely distinct, for there are cases in which a histiocytic nasal lesion has been followed by generalized polyarteritis. ${ }^{1}$

Recently I. Vathy and A. Sáfrán have reported another case of malignant granuloma. ${ }^{6}$ The patient was a 28 -yearold Hungarian man, and the condition started as an area of

\footnotetext{
1 Friedmann, I., Proceedings of the Royal Society of Medicine, 1964, 57, 289.

: Stewart, J. P., Fournal of Laryngology, 1933, 48, 657.

3 Wegener, F., Beiträge zur Pathologischen Anatomie, 1939, 102, 36.

s Godman, G. C., and Churg, J., Archives of Pathology, 1954, 58,

- Vathy, I., and Sáfrán, A., fournal of Laryngology and Otology, 1969, 83, 261 .

Ardouin, A. P., Proceedings of the Royal Society of Medicine, 1964,

57, 299.
}

redness and induration around the root of the nose. There followed relentless destruction of the skin, bones of the face, and the eyes. Histologically the lesion conformed to the histiocytic type of granuloma described by Stewart, but there were also scattered giant cells and some local periarteritis. There was no clinical evidence of generalized disease ; in particular the lungs and kidneys were clear. Death occurred after four months, but no necropsy was performed.

The aetiology of malignant granuloma is unknown. Despite its malignant course there is no evidence that it is a neoplastic process. Indeed, it must be distinguished from reticulum-cell sarcoma and lymphosarcoma of the nose, which may produce a similar clinical picture but differ both histologically and in the development of distant metastases. Since Wegener's granulomatosis is related to polyarteritis nodosa, malignant granuloma has been ascribed to an autoimmune process, ${ }^{1}$ but the evidence is not convincing. Treatment is also unsatisfactory. Antibiotics are of no use, but some cases, especially of the histiocytic type, respond well to radiotherapy. ${ }^{7}$ Adrenal corticosteroids are of value in Wegener's granulomatosis. $^{5}$ Recently two cases of malignant granuloma are reported to have responded to the antimetabolites 6-mercaptopurine and azathioprine. ${ }^{8}$ In Vathy and Sáfrán's case corticosteroids were given only late in the disease, while radiotherapy was not tried. As quite a few cases have survived for years after radiotherapy, ${ }^{7}$ it is advisable to use it as soon as the condition is diagnosed. But until the nature of this mysterious and devastating disease is understood the therapeutic approach can be only empirical.

\section{Accident Surgery}

Accidents are prominent as a cause of disability and death at several different periods of life. As they have always been, they remain an enormous hazard to people aged over 75. But in the first year of life too they cause more deaths than they will do until the seventh decade is reached. For people under 35 in general they are the largest single cause of death, such is their high frequency now in contrast to the diminution of formerly mortal diseases. The startling effect of motor-vehicle accidents is perhaps too little known. In the decade of life 15-24 they cause nearly three times as many deaths as they do in the subsequent decade and nearly four times as many as in the previous decade. Clearly the roads are imposing their own kind of natural or unnatural selection.

Because of this epidemic of violence-not all of it accidental-hospitals have had to increase their facilities for accident surgery, and at the same time the growth of a separate specialty is becoming evident. To meet its needs a new journal entitled Injury has appeared with a strong team from Birmingham in the editorial front line and supporters from the armed Forces as well as many centres with special experience of this work. An editorial article introducing this first issue is headed "Not Another Journal!" But if subsequent issues live up to the promise of the first the apologetic sigh will quickly be drowned in praises for what promises to be an informative publication. Published by John Wright \& Sons, its price at $£ 44$ s. for four issues a year is good value and sets an example to those expensive and needless journals criticized in these columns last week. ${ }^{1}$

1 British Medical fournsl, 1969, 3, 189. 